\title{
Estrategias de marketing digital en la promoción de Marca Ciudad
}

FECHA DE RECEPCIÓN: 17 de enero FECHA DE APROBACIÓN: 1 de febrero Pp. 59-72

\section{Resumen}

El propósito de este estudio es el diseño de estrategias de marketing digital para la promoción de Marca Ciudad. Esta investigación teórica está fudamentada en los autores García (2010), Capurro (2006), Montiel (2002), Porter (2012), Flórez (2012), entre otros. La metodología es cualitativa; se realizaron entrevistas estructuradas y semiestructuradas, además de un trabajo de observación participante a los turistas. Con los productos resultados de la investigación, es decir, un manual o guía de marketing y la elaboración de un sistema digital de aplicación móvil y portal web, se busca generar presencia a un clic de distancia en un mundo de comunicación sin fronteras y avanzar en la competitividad de la ciudad.

\section{Palabras clave}

Marca ciudad, marketing digital, competitividad, innovación, turista, promoción.

\section{David Albeiro Andrade Yejas}

PhD (c) Ciencias, Mención; Gerencia-Universidad Rafael Belloso Chacin (Maracaibo - Venezuela).

Magíster en Software Libre-Universidad Autónoma de Bucaramanga.

Docente IE Rafael Valle Meza-Valledupar. 


\title{
Digital Marketing Strategies in the Promotion of the City Brand
}

\begin{abstract}
Abstrac. This study aims at designing digital marketing strategies to promote the city brand including a marketing manual or guide and the elaboration of a digital system of mobile and web application. This research study was based on the theories of Garcia (2010), Capurro (2006), Montiel (2002), Porter (2012), Florez (2012), and others. Its methodology was qualitative; structured and semi- structured interviews were made, as well as direct observation of tourists. As a consequence, a digital marketing guide and digital applications were designed, representing only one click to access a non-barrier world and to improve the city competitiveness.
\end{abstract}

Key words. City Brand, digital marketing, competitiveness, innovation, tourist, promotion.

\section{Estratégies de marketing digital pour la promotion des villes}

Resumé. L'objet de cette étude consiste en la création de stratégies de marketing digital pour la promotion de marque-ville incluant un guide marketing, l'élaboration d'un système digital d'application mobile et de portail web. Cette investigation théorique repose sur différentes investigations d'auteurs tels que García (2010), Capurro (2006), Montiel (2002), Porter (2012), Flórez (2012), entre autres. La méthodologie utilisée est de type qualitative, incluant des entretiens structurés et semi-structurés ainsi qu'une observation participative des touristes. Les résultats de cette investigation sont la publication d'un guide de marketing digital et la création d'applications digitales permettant à l'utilisateur de se trouver à un simple clic de distance d'un monde de communication sans frontières et pouvoir avancer vers une compétitivité accrue des villes.

Mots clefs. Marque-ville, marketing digital, compétitivité, innovation, touriste, promotion.

\section{Estratégias de marketing digital na promoção da marca cidade}

Resumo. O objetivo deste estudo foi traçar estratégias de marketing digital para a marca cidade que incluem um manual ou guia de marketing e a elaboração de um sistema digital de uma aplicação móvel e portal web; esta investigação teórica foi baseada em Garcia (2010), Capurro (2006), Montiel (2002), Porter (2012), Flórez (2012), entre outros autores. A metodologia foi qualitativa, entrevistas semiestruturadas e estruturadas foram realizadas, além de observação participante aos turistas. De acordo com a orientação acima se obtém um guia de marketing digital e algumas aplicações digitais; que geram presença a um clique de distância em um mundo de comunicação sem fronteiras e promover a competitividade da cidade.

Palabras chave. Marca Cidade, marketing digital, competitividade, inovação, turista, promoção. 


\section{Introducción}

$\mathrm{E}$ n este trabajo se diseñaron estrategias de marketing digital de un territorio, a través de la promoción de la Marca Ciudad, que incluyen un manual o guía de marketing y la elaboración de un sistema digital de aplicación móvil y portal web. El uso de las Tecnologías de la Información y las Comunicaciones (TIC), se ha masificado dentro del ámbito del turismo, convirtiéndose en herramienta fundamental y en un aliado para la conquista de turistas para los diferentes destinos que se promocionan a través de aplicaciones móviles y de portales web o website.

Cada vez más, las entidades turísticas o las empresas recurren a las TIC, en particular Internet, como medio para promocionar sus productos y servicios turísticos. Las nuevas tecnologías han cambiado el concepto de vida de personas en cuanto a la consulta de precio y rapidez de información de los diferentes servicios turísticos, lo que obliga a las ciudades a venderse como un producto, es así como surge el concepto que García manifiesta: «El marketing de ciudades o city marketing puede definirse, por consiguiente, como una política activa integrada por un conjunto de actividades orientadas, por una parte, a identificar y determinar las necesidades de sus diferentes públicos, reales y potenciales; $y$, por otra parte, a desarrollar una serie de productos y servicios en la ciudad para satisfacer dichas necesidades, creando y potenciando su demanda» (García, 2010).

El mismo autor expresa, que desde finales del siglo $X X$, se ha producido un cambio fundamental en las ciudades impulsado por los cambios socioeconómicos, la globalización de la economía y la necesidad de diversificar la base económica, buscando nuevas oportunidades en el sector de servicios, que sitúa a las ciudades en un entorno de competencia. Por su parte, Michael Porter, economista estadounidense, profesor de la Escuela de Negocios de Harvard y reconocido como la principal autoridad mundial en materia de competitividad, expresó que "las ciudades necesitan un pensamiento y un programa de competitividad para que la equidad sea una realidad entre los ciudadanos» (Porter, 2012). Los países más desarrollados deben enfocarse en competir en los intangibles, estos son: marca, calidad, diseño, tecnología, servicio postventa, capacidad innovadora y excelencia en la gestión.

Pensando en la capacidad innovadora, este concepto toma una trascendencia especial en la época actual, al admitir que para muchas organizaciones se convierte en un elemento diferencial de competitividad (Porter, 2012). Es de mucha importancia conocer en detalle la relación que existe entre el gobierno, la innovación y las tecnologías, si se toma como punto de partida la base sobre la cual la competitividad debe ser entendida como la capacidad que tiene una entidad, pública o particular, lucrativa o no, de alcanzar y conservar ventajas comparativas que le faciliten alcanzar, mantener y mejorar una expresa posición en el contexto socioeconómico. Actualmente, este en un escenario que denota un medio urbano con una demanda gradual de eficiencia, desarrollo sostenible, calidad de vida y gestión eficiente de los recursos; las administraciones públicas deben plantear una revolución en los prototipos de gestión de las ciudades. En este aspecto, el aprovechamiento de las TIC se hace necesario.

Por ello, hoy en día la industria del turismo no es ajena al mundo de las TIC; cuando apareció la World Wide Web, el uso de Internet se limitaba a la consulta de noticias, revisión del correo electrónico y una compra puntual 
de productos y servicios. Posteriormente, la red se popularizó y esto conllevó a que aparecieran nuevas herramientas para la elaboración y difusión de contenidos de manera sencilla. Utilidades y servicios que se sustentan en una base de datos, la cual puede ser modificada por los usuarios, ya sea en su contenido o en su forma de presentación. A medida que el Internet siguió avanzando, el turista fue adoptando el rol de productor de contenidos y empezó a subir fotos, videos y comentarios de viaje -en Facebook, Instagram, Twitter, You Tube-, incluso hace recomendaciones de los sitios o lugares que visita y del servicio que recibe.
Internet se puede considerar la herramienta perfecta para eliminar las distancias, al permitir que la información contenida en la red sea compartida por cualquier persona en cualquier parte del mundo, a través de las herramientas computacionales existentes, como son: páginas web, aplicaciones móviles, foros, redes sociales, correo electrónico, etc. Se puede decir, que más que una tecnología, es un medio de comunicación sin fronteras indispensable si se realiza turismo de: placer, negocios, estudios, científico, trabajo, cultura, religioso, gastronómico, salud, etc.

\section{Marco teórico y conceptual}

E

n esta era del conocimiento, los principios de ventajas competitivas, paulatinamente dependerán de la innovación apoyada en este. Es importante saber que no hay otra opción para prosperar que no sea convertir el aprendizaje y la generación de ideas en el factor más relevante, orientado en la innovación tecnológica y científica, por medio de la investigación y desarrollo. Lo anterior necesita de una sólida base tecnológica y científica. El gurú de la economía, Joseph Alois Schumpeter, fue quien primero destacó la relevancia de los fenómenos tecnológicos en el crecimiento económico, esto mejoraría con el impulso al turismo como unos de los motores de desarrollo.

\subsection{Marketing digital}

El marketing se ha integrado en la estrategia de las empresas hasta formar parte indisoluble de su ADN, como organizaciones que compiten en el mercado para ofrecer valor a sus clientes. La función del marketing se ha extendido a todas las organizaciones y empresas, grandes, medianas o pequeñas. Casi todas «hacen marketing» aunque en ocasiones no lo sepan o lo hagan de forma inconsciente o rudimentaria (Marketing FCA, 2009).

Para las empresas, las reglas de juego del mercado cambian rápidamente y «lo digital lo está cambiando todo» (Flórez, 2012). Es de notar que no sólo se trata de la tecnología, sino también de los cambios en la actitud y en el comportamiento de los consumidores, quienes se motivan por el uso de las nuevas tecnologías. Así mismo, el universo digital se extiende en la sociedad y genera nuevos estilos de vida y modernos hábitos de consumo.

Existe una sociedad moderna en la que lo real y lo virtual, lo analógico y lo digital, conviven y se mezclan generando una nueva realidad: «lo virtual es real y lo real es también virtual» (Marketing FCA, 2009). Esto lleva al principal cambio de este mundo digital, y es que se puede estar conectado en todo momento y en cualquier lugar. En ese novedoso universo, emerge y se desarrolla imparablemente el llamado «marketing digital». 
Un reflejo de que el marketing ya no es lo que era, son los innumerables y de recientes conceptos, términos y herramientas que se han introducido en el lenguaje del marketing digital: posicionamiento orgánico, Search Engine Optimization (SEO), keywords, inbound, redes sociales, outbound, SEM O mercadotecnia en buscadores web, AdWords, Display Ads, Facebook Ads, entre otras. La lista es interminable y estos son solo algunos de los nuevos términos, pero el inventario crece sin descanso generando ansiedad y desazón ante una reciente realidad que parece que se escapa o que únicamente es entendida por los expertos; sin embargo, pero es necesario un marketing novedoso como verán a continuación.

¿Por qué se debe utilizar una estrategia digital para la gestión del marketing? Hoy en día, las organizaciones tienen inmensas oportunidades en los medios digitales, desde una empresa de base TIC, hasta negocios

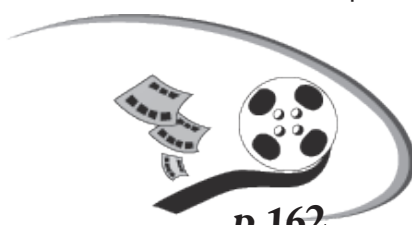
tradicionales. En este sentido todas pueden aprovechar las oportunidades digitales (Adisar Consulting, 2014). Es de resaltar que una estrategia digital proporciona la dirección y unos pasos medibles sobre cómo usar los medios y las técnicas para conseguir la visión y los respectivos objetivos de la empresa. Igualmente, existen muchas razones para utilizar este tipo de estrategia:

- El uso de una estrategia digital permite transformar los datos en inteligencia de mercado, tanto de los clientes como de la competencia, y es claro que la información es necesaria para competir en el mercado (Ideas y proyectos de consultoría, 2012).

- Las estrategias digitales le permiten a la empresa mejorar la relación con los clientes y ver de qué manera interactúan con la marca.
- Hoy en día, el cliente está cada vez más formado e informado en el uso de los medios digitales, y el mercado de las TIC crece sin parar.

- No solo es tener un producto digital, también hay que saberlo enfocar para poderlo vender.

\subsection{Marca País}

Es de singular importancia conocer la experiencia y los resultados que el uso del concepto de marca ha hecho en diferentes países, ciudades y regiones del mundo, para lo cual es conveniente hacer el siguiente recorrido conceptual en asuntos referentes al tema. La marca país refleja una visión holística de la imagen de un territorio que se refuerza y se enriquece con la inversión en comunicación del país de origen, hacia el resto del mundo; los constructores son empresas, marcas líderes y referentes de la sociedad, se utilizan para representar los elementos diferenciadores de un país con respecto a otro.

El término Marca País o country brand nació de la necesidad de los sectores empresariales y de los gobiernos por generar una identidad propia frente a los mercados internacionales. La estrategia de posicionamiento de un país en particular se enmarca en el objetivo de capitalizar el origen de los productos, las empresas y las personas, en los mercados globales (Echeverry, 2015). Continúa dicien-do: «mientras la imagen de un país es la percepción que tienen los consumidores directos, indirectos, reales y potenciales de los países; y es equivalente a la suma de todos los elementos que componen el país, más los que se generan para comunicar sus características; esas percepciones contienen connotaciones diferenciadoras. Todos los países son diferentes, así compartan elementos comunes». 
La Marca País logra hacer visible el marketing territorial, integra los productos, organizaciones e imagen que representa una nación (Echeverry, 2015). Esta refleja una identidad, que se convierte en la esencia de la existencia de la empresa o producto, y enmarca de principio a fin todos los objetivos y las metas, tratando de crear un estilo propio que le permita posicionar su nombre en el mercado y consolidar una reputación y reconocimiento a partir de características propias e inconfundibles.

El concepto de marca es una herramienta fundamental para que países, ciudades y regiones, puedan promocionarse en un mundo que día a día se hace más pequeño, y en el cual los diferentes sitios compiten como destinos para el turismo, para las inversiones y para los compradores de sus productos y servicios (Andrade, 2015). Enmarcado en ese contexto competitivo, casi todos los lugares del planeta están buscando cómo promocionar su cultura, sus atractivos y sus exportaciones por sí mismos.

\subsubsection{Experiencias de Marca País}

Distintos países o regiones siempre han sido asociados con una competencia específica: Chile y sus vinos, Francia y sus quesos, Suiza y sus relojes, Italia y su pasta, Australia y el turismo, entre otros. Pero en años recientes se ha observado una investigación en la que se señala que los consumidores, en distintos países, responden de maneras diferentes a las características del país de origen, y que estas respuestas pueden variar en el tiempo (Deshpandé, 2007). A continuación, se exponen algunos casos.
FutureBrand es una compañía líder en investigación sobre el ranking de Marca País. Tiene a su cargo las mediciones del Country Brand Index desde el año 2005. Para la organización, la Marca País representa variables como: los atractivos, la geografía, la infraestructura, la autenticidad, entre otros; es dedcir, que permiten ubicar a los países en los puntos donde son más fuertes o representan una ventaja competitiva (Echeverry, 2015).

\section{El Country Brand Report} América Latina, analiza la fortaleza de las marcas país de la región, sobre 21 naciones; está basado en un estudio cuantitativo e información

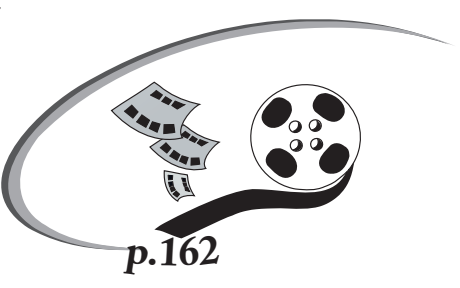
provista por formadores de opinión y viajeros frecuentes por «negocioso placen» externos a Latinoamérica (FutureBrand, 2016). En ese estudio global, Country Brand Index analiza la percepción y asociaciones que generan los países, de la misma manera en que se mide la percepción sobre marcas de consumo y marcas corporativas.

En lo que respecta a Latinoamérica, se siente mayor decisión y acciones más conscientes con relación a la importancia de trabajar la Marca País. Esto se evidencia en la profesionalización de la disciplina, el creciente debate sobre el tema, la proliferación de iniciativas marcarias vinculadas a la Marca País, marcas turísticas, sectoriales y denominaciones de origen, así como en la renovación de estrategias e identidades de las marcas país de la región en los últimos años (FutureBrand, 2016). El estudio de FutureBrand, Country Brand Report América Latina 2015/16, evalua atributos como: made In -país de origen-, turismo, patrimonio y cultura, calidad de vida, aptitud para los negocios y sistema de valores (Tabla 1). 
Tabla 1. Ranking de países latinoamericanos

\begin{tabular}{|c|c|c|}
\hline 26 & ARGENTINA & \\
\hline 30 & MÉXICO & +3 \\
\hline 40 & CHILE & \\
\hline 5 & PERÚ & \\
\hline $6 \theta$ & COSTA RICA & -3 \\
\hline $7 \Theta$ & PANAMÁ & +1 \\
\hline $8 \theta$ & CUBA & +4 \\
\hline $9 \theta$ & PUERTO RICO & $\overline{+1}$ \\
\hline 100 & URUGUAY & -3 \\
\hline
\end{tabular}

Fuente. http://www.cbramericalatina.com

\subsubsection{Creación y desarrollo de la Marca Ciudad}

Las dos premisas de las que parte la creación y desarrollo de la Marca Ciudad, son: la primera, que la ciudad toma su forma, contenido y significado en la mente de las personas; y la segunda, que la gente conoce y entiende la ciudad a través de sus propias percepciones, y las procesa a través de la imagen que la esta ofrece. Para Saez, Mediano y De Eizagarate (2011) «este es el mismo proceso que se sigue en la formación de imágenes de bienes, servicios y empresas, y que ha sido gestionado de manera exitosa como marcas durante mucho tiempo».

El marketing introduce el concepto de mercado en las ciudades, lo que lleva a considerar un cambio en la forma de pensar y considerar a las metrópolis, a los inversores y a sus visitantes. Dicho concepto implica un viraje de orientación y una nueva filosofía de gestión, según Saez (2010) «al considerar

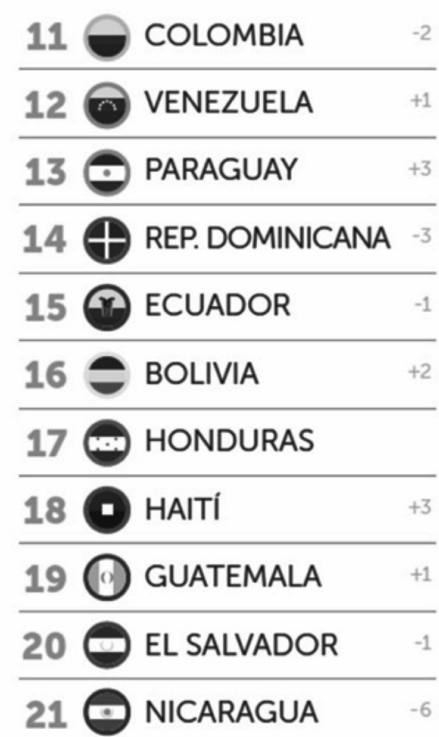

que la ciudad se encuentra inmersa en un proceso de intercambio, que da lugar a las transacciones entre los servicios y atracciones de la ciudad y sus diferentes públicos objetivo: ciudadanos, inversores, empresas o turistas». Como resultado se produce una estrecha red de relación de satisfacción, en donde el ciudadano, como cliente, es lo más importante.

Como cualquier producto o artículo de consumo, las características propias de la ciudad son los elementos diferenciadores y de competencia con las demás. Uno de los atributos de mayor importancia es la imagen de marca, y junto con esta, la marca registrada. Este activo intangible no se puede administrar al azar, sino que debe obedecer a un completo plan de marketing estratégico y es ahí donde adquieren una vital trascendencia las estrategias de comunicación. «El plan de marketing estratégico ha de contemplar la definición de una imagen fuerte, diferente, reconocible, coherente $y$, sobre todo, creadora de una opinión favorable entre los diferentes públicos a los que se dirige» (Saez, 2010). 
El motivo que mueve a la construcción de una Marca Ciudad, se fundamenta esencialmente en promover la ciudad «como destino turístico, centro de negocios y lugar de residencia. Pero construir una Marca Ciudad no es solamente recrear una identidad visual» (Capurro, 2006). Es común ver eslóganes como «Das Neue Berlín»; «Edimburgo: Capital de Inspiración»; Ámsterdam, lanzó recientemente una campaña de branding centrada en el eslogan «l Ámsterdam»; y Londres, por su parte, se ha convertido en «Totally London».

Cada vez más, las marcas de las ciudades son consideradas un activo importante para el desarrollo urbano; además, son la herramienta efectiva por medio de la cual se diferencian y mejoran su posicionamiento. Ciudades, en diferentes partes del mundo, utilizan varias vías para promocionarse en audiencias relevantes como inversores, visitantes y residentes, y en sus esfuerzos incluyen logros impactantes y eslóganes cautivadores que protagonizan las páginas web de bienvenida y las campañas de publicidad en los medios nacionales e internacionales.

\subsection{Uso de las TIC en el turismo}

La informática y las telecomunicaciones pueden actuar en el producto turístico como oferta, con una mejora y modernización del modelo tradicional y con la incorporación de nuevos productos. En la difusión del patrimonio turístico, mejorando su promoción y comercialización, elementos que son claves en el valor añadido y según (Montiel, 2002), la «ayuda a la empresa turística como a cualquier otra persona comunicándola con sus entornos afines y proporcionándole las ventajas inmediatas de la automatización de tareas habituales».

\subsection{Mercado móvil en Latinoamérica}

Diferentes estudios indican la fragmentación del mercado digital en Latinoamérica, lo que implica la necesidad de que las marcas deben estar presentes en las diferentes plataformas, especialmente en los smartphones y tablets que han desplazado a los portátiles y notebooks. Estos mercados están registrando un aumento exponencial, de la misma manera que las herramientas de publicidad disponibles para las empresas en ese mundo online. Según la Organización de Operadores Móviles (GSM), y compañías relacionadas con el sistema de telefonía móvil, hay 328 millones de usuarios móviles individuales en Latinoamérica. Para finales de 2014 habría 341 millones de usuarios móviles latinoamericanos, y para 2017, habrá 374 millones (Ardila, 2016).

En el ámbito de la publicidad móvil, las oportunidades también siguen creciendo y se espera que este segmento crezca en la actualidad mucho más que cualquier otro, especialmente si se considera que América Latina se encuentra densamente poblada por jóvenes de entre 15 y 34 años, representando este grupo más del $50 \%$ de todos los usuarios digitales en América Latina (Castrejon, 2016). Las marcas y las agencias de publicidad enfrentan el desafío de entender el mundo digital, estar al día con todos los avances de la tecnología y comprender cómo impactan en los cambios de paradigmas el consumo de las personas. 


\section{Marco metodológico}

E

diseño metodológico de la presente investigación está soportada en un enfoque cualitativo. Para Hernández, Fernández y Baptista (2010) este se selecciona cuando se busca comprender la perspectiva de los participantes -individuos o grupos pequeños de personas- acerca de los fenómenos que los rodean, profundizar en sus experiencias, perspectivas, opiniones y significados; es decir, la forma en que los participantes perciben subjetivamente su realidad, pues dado el objeto de estudio, se necesita establecer información en profundidad sobre aspectos subjetivos, percepciones y emociones de los turistas cuando visitan la ciudad de Valledupar o se proponen visitarla. Seis elementos resultan fundamentales para plantear un problema cualitativo: objetivos de investigación, preguntas de investigación, justificación, viabilidad, evaluación de las deficiencias en el conocimiento del problema y definición inicial del ambiente o contexto.

La investigación aprovechó el uso de Internet en los turistas mediante la conexión a través de los smartphones, para indagar si mediante una aplicación móvil y portal web se promocionaría a la ciudad de Valledupar como marca. Por lo tanto, una fuente de información primaria importante fue la suministrada por los turistas, además de las oficinas que promueven el turismo en la dicha ciudad y por los prestadores de servicios como hoteles, restaurantes, casinos, etc.
Para obtener información básica sobre los sitios turísticos de la zona objeto de este estudio, se realizó un recorrido por ella. La visita se llevó a cabo con entrevistas estructuradas y semiestructuradas, a turistas y propietarios de negocios; además, se combinó con la técnica de la observación participante, que implica tener en cuenta la existencia del observador, su subjetividad y reciprocidad en el acto de observar. El énfasis, en estos momentos, se pone en la diferencia; sujetos de diferentes ambientes o estratos sociales son también capaces de tener sensaciones, manifestar sentimientos, formular argumentaciones lógicas y comunicarse. Hay diferencias entre los grupos, entre las culturas, diversidad de historias, y también hay un interés por la búsqueda del sentido, que se presenta en las experiencias subjetivas y afectivas de las personas. Predomina la comprensión de la complejidad de los fenómenos, en una aproximación hermenéutica y no su explicación causal (Hernández, Fernández, y Baptista, 2010).

\subsection{Actividades}

A continuación se describen las actividades desarrolladas en la ejecución de este proyecto (Figura 1): 
Figura 1. Diseño metodológico

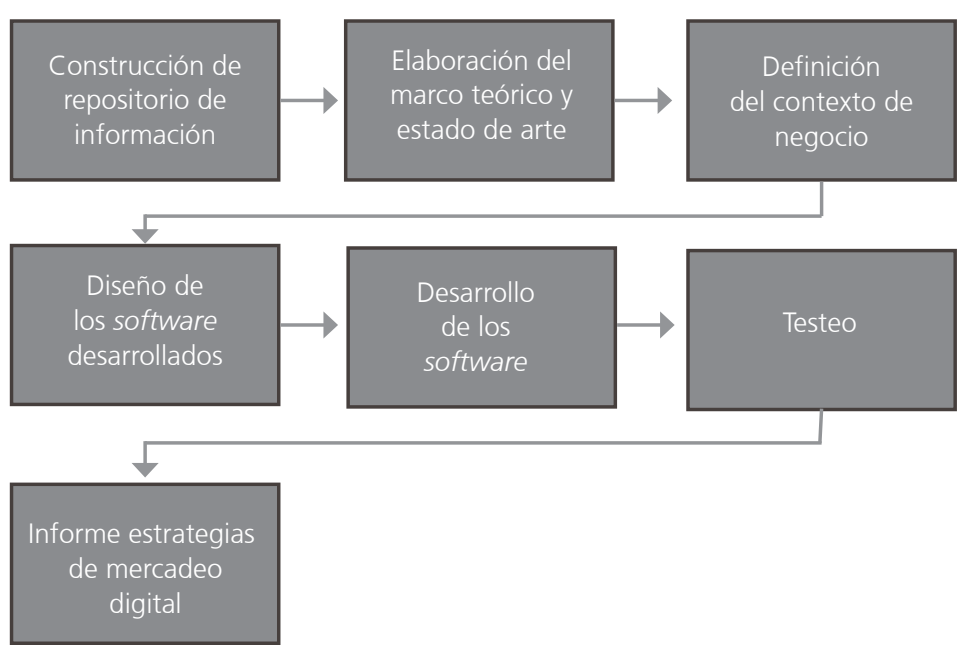

Fuente. Elaboración propia del autor.

- Construcción de repositorio de información. Esta actividad tuvo como propósito obtener información de las principales fuentes de datos disponibles, como Google Scholar, Scopus, IEEE, UOC y recuperar los artículos de interés para el desarrollo del proyecto.

- Elaboración del marco teórico y del estado del arte. El desarrollo del marco teórico permitió esbozar una visión clara de los proyectos, que en materia de city branding, han sido desarrollados a nivel nacional e internacional. Se analizaron las técnicas, metodologías y estrategias usadas por los territorios que han logrado concretar casos exitosos en el tema.

- Definición del contexto de negocios. El modelo de negocio Canvas permitió identificar los actores participantes y las relaciones entre ellos.
- Diseño de los software desarrollados. Primero se realizan las validaciones en campo, con entrevistas; se realizan los mockups y wireframes, además de los diseños de las aplicaciones para teléfono móvil y para website.

- Desarrollo del software. En esta etapa de desarrollo y usando métodos SCRUM iterativo e incremental, se crearon los códigos de los principales componentes y artefactos de las aplicaciones establecidas en la parte de diseño.

- Testeo. En esta fase se realizaron todas las pruebas necesarias a las aplicaciones desarrolladas.

- Informe estrategias digitales. Se complementaron las aplicaciones desarrolladas con un manual de marketing digital. 


\section{Resultados}

$\mathbf{R}$ epositorio de investigación: de manera estratégica, resultó acertado tomar todo el tiempo necesario en esta etapa, que en sí, no es de ingeniería pura, pero permitió contextualizar con el entorno, aterrizar los conceptos, conocer de primera mano las necesidades de los usuarios y clientes potenciales, además de explorar los criterios con respecto al manejo de la marca que tenía la administración pública municipal.

\subsection{Estrategias de Marketing digital}

- Crear identidad digital. La identidad digital es el rastro que cada usuario de Internet deja en la red como resultado de su interrelación con otros usuarios o con la generación de contenidos. Una identidad digital no está definida a priori y se va conformando con la participación, directa o inferida en las comunidades y servicios de Internet. Se recomienda que la marca cree su identidad digital a partir de la participación en diferentes redes sociales y que sean consecuentes con el producto y servicio.

- Trabajar la Ley de enfoque. Para Trout y Ries, (2012) «el principio más poderoso en Marketing es poseer una palabra en la mente de los clientes». Una marca puede llegar a tener un éxito increíble si logra apropiarse de una palabra en la mente del cliente.

- Posicionarse en buscadores. Las marcas deben buscar estrategias para posicionarse en los buscadores, de tal forma que estén a la vista de su público objetivo y puedan ser visualizados rápidamente.

- Implementación de SEO. Se trata de las acciones requeridas para lograr que un sitio web de la marca, se posicione entre los primeros resultados en un buscador de forma orgánica/natural con los términos que describen su producto o servicio.

- Inbound Marketing. Son todas las estrategias de Marketing en donde "No tienes que pagar". Según explica Internet República en su blog, el inbound marketing se basa en tres pilares fundamentales: SEO, marketing de contenidos y social media marketing. Ya sea en redes sociales como Twitter, en Facebook con la creación de páginas empresariales, YouTube.

- Outbound Marketing. Para Carballo, (2011) «son las estrategias de Marketing por las cuales pagas para tener un retorno de inversión (conversión)». Está dirigido a obtener clientes por medio de acciones concretas, se enfoca en buscar y «atraer nuevos leads enviando una gran cantidad de mensajes por varios medios para causar diferentes impactos en más personas y lograr así que se conozca el producto» (Carballo, 2011). Se utilizan herramientas como:

- SEM. Se paga por aparecer de primero en una búsqueda. Con Google se tiene AdWords, el cual sirve para SEM y para Display Ads.

- Display Ads. Es toda publicidad que se encuentra en una web, en un banner donde se promociona una marca.

- Anuncios en Youtube. Se encuentran en dos tipos; primero, está el anuncio común insertado en el video. El segundo se refiere a los anuncios al estilo de propagandas que aparecen al inicio de un video. 
- Facebook Ads. Ofrecen dos tipos de anuncios, el anuncio al lado derecho y el anuncio central.

- Twitter. Aquí se puede comprar hashtag, promocionar cuentas por búsqueda o simplemente, aparecer en las sugerencias de cuentas.

Estas recomendaciones deben ser lideradas por un estratega digital, el comunity manager o gestor de la comunidad de Internet, el cual se debe encargar de crear múltiples campañas de educación, promoción, lanzamiento, posicionamiento y mantenimiento de la marca en los nuevos medios y canales digitales de comunicación -website, aplicaciones móviles-. Estar en el ciberespacio no es complicado para ninguna organización, como resultado de una aplicación sensata y eficaz de una estrategia de comunicación con canales web en la plataforma global que sean efectivos, usables y sincronizados con la entidad.

\subsection{Creación y uso de aplicaciones digitales}

Cambiar de paradigma de móviles a PC, en vez de PC a móviles, no sólo prepara para el futuro cercano, en el que desarrollar sitios optimizados para estos dispositivos, no será simplemente un lujo de grandes empresas, sino una necesidad real del mercado en pro de un producto más efectivo y usable.

Crear la website y la aplicación móvil no era sólo cuestión de colocar elementos aquí o allá para que quede bonito, se trataba de construir una oficina virtual de turismo que cupiese organizadamente dentro de estas. Además, debía contar con interacción directa del usuario que genera una cualidad única, por medio de críticas, sugerencias a servicios, comentarios y productos turísticos, dando a conocer experiencias a través de blogs, vídeos y fotos, y ejerciendo influencia nítida sobre la percepción y decisión de otros clientes. Conjuntamente con las siguientes bondades para el turista: sencillez absoluta, cooperación entre los usuarios, mayor funcionalidad esencial, más rapidez, predicción y personalización de la comunicación, ubicuidad y presencia de información. ¿Qué debería contener?

\subsubsection{Inventario de contenidos}

De acuerdo con la metodología para la elaboración de inventarios de atractivos turísticos del Ministerio de Comercio, Industria y Turismo, los atractivos se clasificaron en patrimonio cultural y sitios naturales, así:

- Recursos naturales.

- Patrimonio cultural material en el área urbana.

- Monumentos.

- Centro histórico.

- Patrimonio cultural en el área rural.

- Patrimonio cultural inmaterial.

A continuación se observan aspectos importantes del producto final, fruto de este trabajo una aplicación móvil multiplataforma (Figura 2). 
Figura 2. Aspectos de la aplicación móvil.
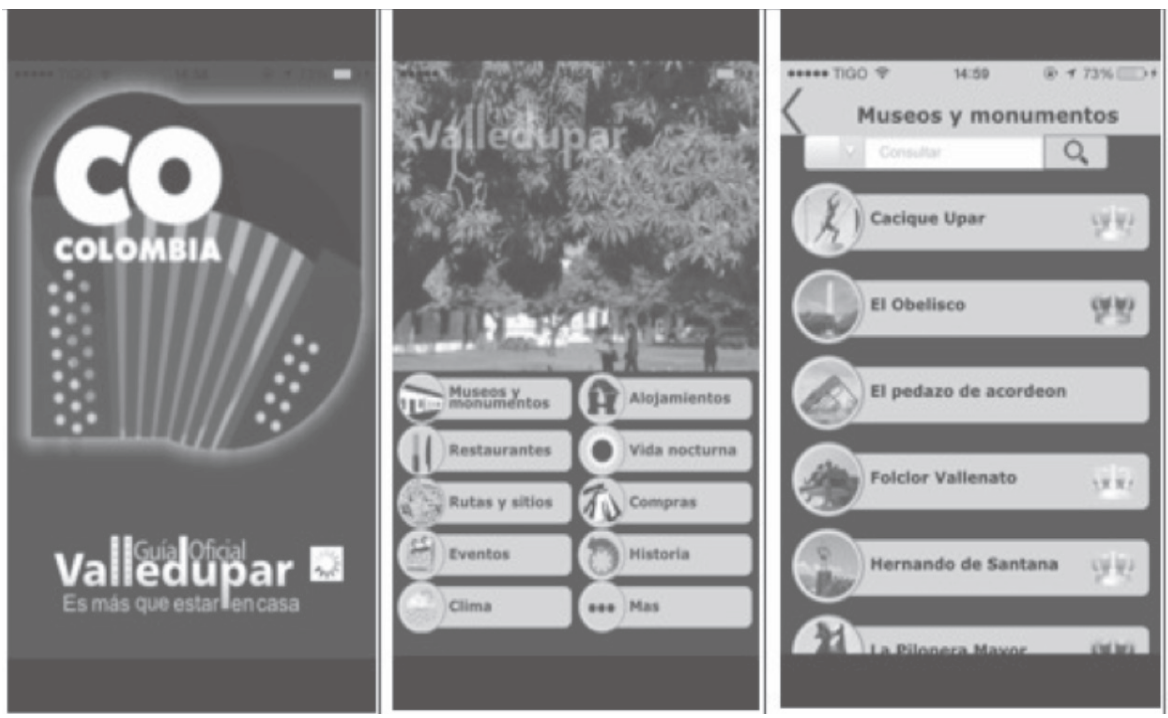

Fuente. Elaboración propia del autor.

\section{Conclusiones}

E

I estudio realizado se focalizó en la ciudad de Valledupar, pero de igual manera se puede extrapolar y servirá de guía a futuros investigadores, además de permitir a los turistas gozar con una oficina de promoción turística en sus manos, donde encontrarán toda la información que necesita sobre qué ver, dónde pernoctar, qué comer, qué comprar; actividades de ocio, eventos, servicios, mapa con los puntos de interés geolocalizados, para llevar al usuario hasta ellos, y la historia de la ciudad.

La competitividad emergente entre destinos turísticos ha puesto de manifiesto la

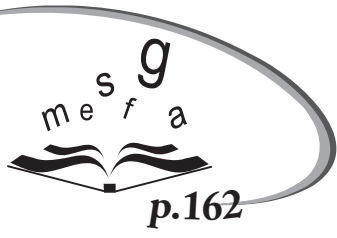
necesidad de enfatizar estrategias de marketing digital, con el fin de crear un valor agregfado intangible para el cliente potencial, encaminadas a crear una imagen favorable de los territorios que se pretenden promocionar.
Se obtiene un beneficio social al tener disponibilidad de una oficina de turismo en su smartphone o en su tablet. Así mismo, en el mejoramiento de la eficiencia en la búsqueda de la información necesaria para el turista, para tomar las decisiones pertinentes. Al no necesitar papel se está protegiendo el medio ambiente; también, se reducen costos económicos de promoción de la Ciudad, al evitar afiches y demás elementos promocionales.

Mejoramiento de la competitividad de la ciudad de Valledupar con la gestión de la marca, así lo entendieron las ciudades que hace unos años se dedicaron a desarrollar marca por medio del City Branding. Lograron así convertirse en las ciudades que son hoy en día. Para alcanzar un buen desarrollo del City Branding, se requiere de bastantes años y del apoyo de todos los involucrados con la ciudad: gobernantes, instituciones, personas del común, etc. 


\section{Referencias bibliográficas}

Adisar Consulting. (2014). Definición de la estrategia digital, empresas de internet y negocios digitales. ADISAR CONSULTING.

Andrade, D. (2015). Aplicaciones digitales con Software libre para City Branding Valledupar. Tesis Maestria, Universidad Autonoma de Bucaramanga.

Ardila, I. (2016). Publicidad \& Mercadeo. Recuperado de:

http://www.revistapym.com.co/ destacados/radiografia-mercado-movillatinoamerica-2014

Capurro, D. (2006). (s.t.) Recuperado de: http://www.lanacion.com.ar/: http://www. lanacion.com.ar/815713-las-ciudadesquieren-convertirse-en-marcas

Castrejon, R. (2016). América Latina Bussines Review. Recuperado de http://www. businessreviewamericalatina.com/ marketing/1042/El-nuevo-

mercado-m\%C3\%B3vil-enLatinoam\%C3\%A9rica-para-el-2016

Deshpandé, R. (2007). El Efecto País. Harvard Bussines Review, 22.

Echeverry, L. (2015). País Marca OBS. Recuperado de: http://paismarca.com/ 2015/09/14/la-marca-pais-el-reflejo-de-laidentidad-cultural-y-empresarial-de-unanacion/

Flórez, C. (2012). Plataforma de Innovación para el sector textil - confección. Medellin: (s.e).
FutureBrand. (2016). Country Brand Report América Latina 2015/16. The Future Brand. (s.c.) (s.e.).

García, J. S. (2010). Marketing para ciudades: las ciudades también se venden, las ciudades también son productos. Pensar la Publicidad, IV(1), 211-226.

Hernández S, R., Fernández C., y Baptista, M. (2010). Metodología de la investigación. Mexico: McGraw Hill.

Ideas y proyectos de consultoría. (2012). Estrategias de Marketing Digital para PYMES. Anetcom.

Marketing FCA. (2009). (s.t). Recuperado de: http://mktfcaunam.blogspot.com.co/

Montiel, M. (2002). Promoción turística en Internet: las TIC como herramientas de divulgación e información. Memorias, IV Congreso Turismo y Tecnologias de la Información y Comunicaciones, Malaga.

Porter, M. (2012). Expogestión. Barranquilla, Colombia.

Saez, L. (2010). El Valor de la Marca Ciudad como elemento diferenciador. Revista de Administración.

Saez, L., Mediano, L., y De Elizagarate, V. (2011). Creación y desarrollo de la Marca Ciudad. Revista de Dirección y Administración de Empresas. 